\title{
Effects of Virtual Reality Programs on Proprioception and Instability of Functional Ankle Instability
}

\begin{abstract}
We investigated the effects of a Virtual reality(VR) program on the proprioception and instability of functional ankle instability(FAl) patients. Among the 20s old individuals who lived in Republic of Korea; 20 people were selected through a public participation process. The 20 participants were then randomly divided into two groups, one of strength exercise $(n=10)$ and one of balance exercise $(n=10)$. Of the patients who had experienced an ankle sprain in the past and currently felt ankle instability, those who recorded 24 points or less on the Cumberland ankle instability tool(CAIT) were determined to have FAl. We selected the strength exercise and balance exercise in the Nintendo Wii Fit Plus as a VR program. The subjects used the program on the Wii Balance Board three times per week for 20 minutes and total 10-minute warm-up and down for four weeks. Proprioception and CAIT of the balance exercise group were improved significantly after the exercise compared to before the exercise $(p<.05)$. Authors recommend that the balance exercise in the VR program be used as an aid for physical therapeutic intervention.
\end{abstract}

Key words: Virtual Reality; Nintendo Wii Fit Plus; Functional Ankle Instability; Propriocetion; CAIT

\author{
Ki Jong Kimª, Hyun ju Jun ${ }^{a}$ \\ ${ }^{a}$ Cheongam College, Suncheon, Korea \\ Received : 2 June 2015 \\ Revised : 12 July 2015 \\ Accepted : 17 August 2015 \\ Address for correspondence \\ Hyun ju Jun, PT. Ph.D \\ Department of Physical Therapy, \\ Cheongam College, 1641 Noksaek-ro, \\ Suncheon, Korea \\ Tel: 82-61-740-7339 \\ E-mail: juju98jhj@daum.net
}

\section{INTRODUCTION}

In a virtual reality(VR) program, the computer provides reality like three dimensional environments for interaction with users; the users then feel like the environments are real(1). The software used for VR exercises enables the users to establish the intensity and period of exercise and to experience virtual environments in an amiable state with their activities safely controlled(2).

A VR program is particularly useful in physical therapy because its structure can properly motivate patients who need repetitive motor learning and feedback(3). Through direct feedback, a VR program can provide satisfaction to the users and help them develop a considerable sense of balance(4).

The human ankle joint has an extremely stable structure anatomically owing to the tibia, talar dome, ligament constitution, joint capsule, tendon, and traversing direction of the sinews. However, a lateral ankle sprain commonly occurs because the medial malleolus is higher than the lateral malleolus and cannot be properly protected. While there is a strong deltoid ligament inside the ankle for protection and the lateral malleolus helps to block excessive eversion, no such protection exists on the lateral ankle(5,6). A lateral ankle sprain could develop to be functional ankle instability(FAI).

An FAI can be defined as the subjective sense of intermittent unsteadiness on the ankle(7); the patients then find it hard or unstable to walk on an uneven surface. The patients may suffer short-term dysfunction even with slight acceleration, but in general they can live a life without pain and dysfunction $(8,9)$. However, it is the direct association with ankle sprain that is the biggest risk factor of an FAI(10).

Intensified training of proprioception is one of the interventions to alleviate ankle instability. Proprioception is a type of neuromuscular process needed to maintain posture during body activities(11, 12). Research on proprioception is required for FAI patients because the intense training of 
proprioception improves the reaction velocity of peroneus muscles, sense of body, and postural control(13-17).

In this study we, therefore, investigated the effects of a VR program on the proprioception and instability of FAI patients, identifying whether the program could be used as a physical therapeutic intervention for the symptom.

\section{METHODS}

\section{Subjects}

Among the 20 year old individuals who lived in Gwangju and Suncheon, 20 people were selected through a public participation process. The 20 participants were then randomly divided into two groups, one of strength exercise $(n=10)$ and one of balance exercise $(n=10)$. Of the patients who had experienced an ankle sprain in the past and currently felt ankle instability, those who recorded 24 points or less on the Cumberland ankle instability tool(CAIT) were determined to have FAI. No patient participated in other exercise programs associated with the ankles, and patients were excluded from this study when their ankle pain increased after intervention.

This study was preliminarily approved by the Committee of Clinical Tests at Gwangju Oriental Hospital of Dongshin University(IRB No. DSGOH-019). We explained the aims of the study to the participants and presented a written consent form to secure voluntary participation. The general characteristics of the subjects are shown in Table 1.

\section{VR Program}

All subjects were asked to perform a 5 minute warm up and a 5 minute cool down exercise. We selected the strength exercise and balance exercise in the Nintendo Wii Fit Plus(Nintendo Inc., Kyoto, Japan) as a VR program. The subjects used the program on the Wii Balance Board three times per week for 20 minutes for four weeks(Fig. 1). The strength exercise consisted of lunge, single leg extension, sideways leg lift, single leg twist, and rowing squat, while the balance exercise included soccer heading, ski slalom, tight ropewalk, table tilt, and snowboard slalom. All the exercises in the program were performed under the supervision of a physiotherapist for safety and for maintaining appropriate intensity. The daily exercise program lasted 30 minutes; a subject who could not participate in the program because of an unavoidable reason was asked to do the exercises another day.

\section{Measurement of Ankle Proprioception}

We used a Biodex isokinetic Dynamometer (Biodex Medical Systems, Shirley, NY, USA) to measure proprioception of the ankle. The subjects wore eye masks to minimize visual compensation, and the proprioception was measured in a quiet environment to minimize auditory compensation. A subject was asked to perceive the setup angle of the ankle for 10 seconds and then to recover the previous setup position. The subject pressed the button of the dynamometer when they judged that their ankle reached the setup position. Then, the difference between the previously setup position and the one the subject selected was obtained. The measurements were performed in both the sagittal and horizontal planes. We recorded the absolute value of the mean values obtained from three measurements(18, 19). The pose that the subjects held during the measurements is shown in Figure 2.

\section{Measurement of Ankle Instability}

We used the CAIT(ICC2,10.96) to investigate the degree of ankle instability. CAIT is a self-reported 30 -point tool containing a total of nine items. A subject recording at least 28 points was defined as having a normal ankle, while those with a point score of 24 or less was defined as having FAI. A higher point count indicates greater ankle stability $(20,21)$.

Table 1. General characteristics of study participants

\begin{tabular}{ccc}
\hline & Group A & Group B \\
\hline Sex(male/female) & $2 / 8$ & $2 / 8$ \\
Age(years) & $22.6 \pm 1.4$ & $23.2 \pm 1.0$ \\
Height (cm) & $160.6 \pm 8.7$ & $165.6 \pm 5.9$ \\
Weight (kg) & $55.2 \pm 11.6$ & $54.8 \pm 9.7$ \\
CAIT (score) & $17.7 \pm 6.4$ & $19.2 \pm 5.2$ \\
\hline
\end{tabular}

Numbers indicate mean \pm standard deviation

Group A: Strength exercise group, Group B: Balance exercise group CAIT: Cumberland ankle instability tool 
Table 2 Changes in proprioceptive and ankle instability CAIT

\begin{tabular}{|c|c|c|c|c|c|c|c|}
\hline & \multirow{2}{*}{ Plane } & \multicolumn{3}{|c|}{ Group A } & \multicolumn{3}{|c|}{ Group B } \\
\hline & & Pre-test & Post-test & $p$ & Pre-test & Post-test & $\mathrm{p}$ \\
\hline Proprioceptive $\left(^{\circ}\right.$ ) & Sagittal & $4.4 \pm 2.3$ & $3.1 \pm 1.6$ & 0.100 & $3.4 \pm 1.1$ & $1.8 \pm 1.1$ & $0.000^{*}$ \\
\hline \multirow[t]{2}{*}{ CAIT(Score) } & Horizontal & $2.7 \pm 1.8$ & $2.4 \pm 1.0$ & 0.631 & $3.8 \pm 1.9$ & $2.2 \pm 1.1$ & $0.029^{*}$ \\
\hline & & $18.5 \pm 4.7$ & $20.3 \pm 6.6$ & $0.021^{*}$ & $19.8 \pm 3.2$ & $23.9 \pm 3.5$ & $0.047^{*}$ \\
\hline
\end{tabular}

Numbers indicate mean \pm standard deviation, ${ }^{*} p<0.05$

Group A: Strength exercise group, Group B: Balance exercise group

CAIT: Cumberland ankle instability tool

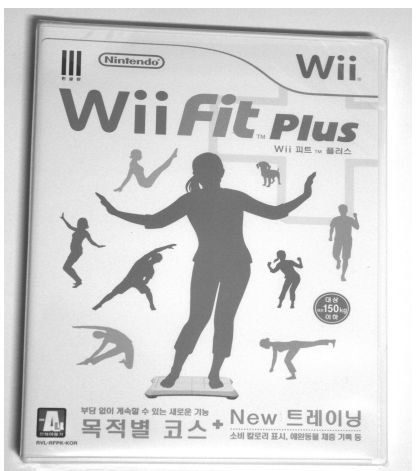

Fig. 1. Nintendo Wii Fit Plus.
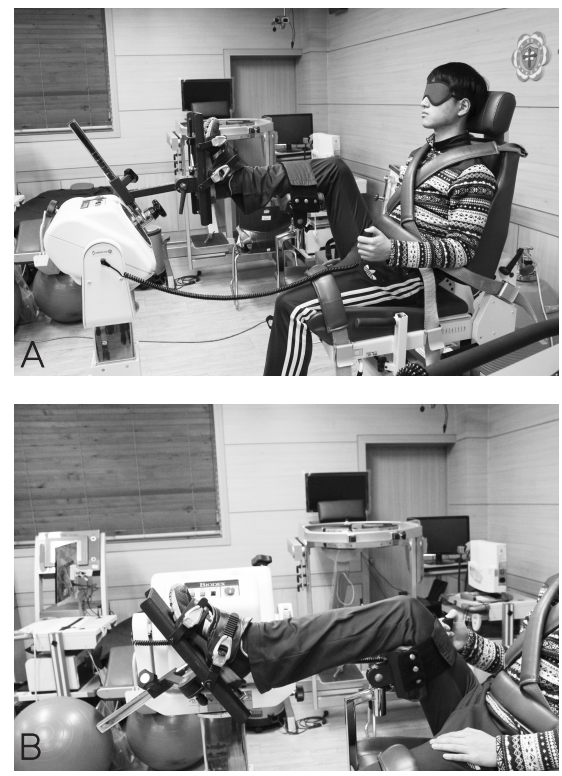

Fig. 2 Measure of ankle proprioception

$$
\text { A: Sagittal plane, B: Horizontal plane }
$$

\section{Data Analysis}

We calculated descriptive statistics quantity of means and standard deviation using the PASW 18.0 statistics program. The One Sample
Kolmogorov Smirnov test was used to investigate the normal distribution of the measurements. The paired samples t-test was performed to analyze significant difference in the results between pre and post exercise. The significance level of the data was set as $\mathrm{p}=.05$.

\section{RESULTS}

The strength exercise group did not show a significance difference between pre and post exercise(p .05$)$ for proprioception in the sagittal plane or the horizontal plane after the VR program; however, proprioception of the balance exercise group declined significantly after the exercise compared to before the exercise $(\mathrm{p}<.05)$ in both planes(Table 2). Post exercise CAIT points of both groups increased significantly after the VR program when compared to the pre exercise points(p<.05)(Table 2).

\section{DISCUSSION}

Proprioception is essential in ankle rehabilitation and an important factor in physical therapy for ankle sprain(22). Gehring et al.(23) stated that understanding the functional movement of the ankle joint is necessary to effectively reduce ankle sprain. Research should focus more on the dynamics of neuromuscular control because neuromuscular control requires proper coordination of somatosensory, vestibular, and visual factors and the proprioception information provided by the receptors in other areas, such as muscles, tendons, joints, and skin. A consistent provision of accurate information of proprioception is important 
for effective feedforward and feedback, preventing ankle sprain even with unexpected ground state or environmental changes. In this context, strengthening proprioception is important in physical therapy for FAI and for normalization of the range of motion(24). The results of this study showed that the group practicing balance exercise through Nintendo Wii Fit Plus improved their proprioception in the sagittal plane and the horizontal plane. Previous studies reported that strengthening proprioception improves the reaction velocity of peroneus muscles and motor sensation and reduced posture sway(14, 15, 16, 17). In a study of runners, there was a significant difference in eversion proprioception between the subjects who experienced ankle sprain and those who did not; unfavorable proprioception affected postural stability and changed reactions for weight support(25). Given the results of such previous researches, the VR program used in this study may induce consistent signal transmission of the afferent and the efferent nerves to contribute to strengthening proprioception through interaction with the central nervous system $(13,26)$. However, we consider that the strength exercise in the VP program may be inappropriate in strengthening proprioception because the strength exercise group did not show a significant difference in proprioception changes in the sagittal and horizontal planes. Thus, the VR program training may be a more effective tool when used as a physical therapeutic interventional aid to improve proprioception for individuals with ankle sprain.

The CAIT is a widely used and reliable tool to assess FAI $(27,28)$. Our results showed that both the strength exercise group and the balance exercise group had a significant increase in CAIT points in the post exercise period compared to pre-exercise. These results are similar to those of a previous study reporting that a six week, dynamic, neuromuscular strengthening exercise resulted in an increase in CAIT points(29) and suggest that the VP program training has a positive effect on FAI. A stable ankle indicates better quality of life with respect to health and less functional limitation(30); thus, increasing the CAIT points via intervention may have considerable significance to reduce FAI.

The results of this study are somewhat limited because our results were not compared to those of existing interventions. Further studies are needed to compare existing physical therapeutic interventions and the VR based intervention.

\section{CONCLUSION}

We identified that the balance exercise in the VR program increased proprioception in the sagittal and the horizontal planes and decreased ankle instability, while the strength exercise did not result in any significant difference in proprioception but reduced ankle instability. We recommend that the balance exercise in the VR program be used as an aid for physical therapeutic intervention.

\section{REFERENCES}

1. Deutsch JE, Borbely M, Filler J, Huhn K, Guarrera-Bowlby P. Use of a low-cost, commercially available gaming console (Wii) for rehabilitation of an adolescent with cerebral palsy. Physical therapy 2008; 88(10): 1196-1207.

2. Yong Joo L, Soon Yin T, Xu D, Thia E, Pei Fen C, Kuah CW, Kong KH. A feasibility study using interactive commercial off-the-shelf computer gaming in upper limb rehabilitation in patients after stroke. Journal of rehabilitation medicine 2010; 42(5): 437-441.

3. Koritnik T, Bajd T, Munih M. Virtual environment for lower-extremities training. Gait \& posture 2008; 27(2): 323-330.

4. Kim SS, Min WK, Kim JH, Lee BH. The Effects of VR-based Wii Fit Yoga on Physical Function in Middle-aged Female LBP Patients. Journal of physical therapy science 2014; 26(4): 549-552.

5. Neumann DA. Kinesiology of The Musculos keletal System: Foundations for rehabilitation, 2nd Ed. St Louis, Missouri. Mosby Elsevier 2010: 591-603

6. Jones MH, Amendola AS. Acute treatment of inversion ankle sprains: immobilization versus functional treatment. Clinical orthopaedics and related research 2007; 455: 169-172.

7. Delahunt E, Coughlan GF, Caulfield B, Nightingale EJ, Lin CW, Hiller CE. Inclusion criteria when investigating insufficiencies in chronic ankle instability. Medicine and science in sports and exercise 2010; 42(11): 2106-2121.

8. Chan KW, Ding BC, Mroczek KJ. Acute and chronic lateral ankle instability in the athlete. Bulletin of the NYU hospital for joint diseases 2011; 69(1): 17-26.

9. Freeman MA. Instability of the foot after injuries to the lateral ligament of the ankle. The Journal of bone and joint surgery British volume 1965; 47(4): 669-677. 
10. Hadadi M, Mousavi ME, Fardipour S, Vameghi R, Mazaheri M. Effect of soft and semirigid ankle orthoses on Star Excursion Balance Test performance in patients with functional ankle instability. Journal of science and medicine in sport / Sports Medicine Australia 2014; 17(4): 430-433.

11. Stillman BC. Making sense of proprioception: the meaning of proprioception, kinaesthesia and related terms. Physiotherapy 2002; 88(11): 667676.

12. Laskowski ER, Newcomer-Aney K, Smith J. Refining rehabilitation with proprioception training: expediting return to play. The Physician and sportsmedicine 1997; 25(10): 89-104.

13. Postle K, Pak D, Smith TO. Effectiveness of proprioceptive exercises for ankle ligament injury in adults: a systematic literature and meta-analysis. Manual therapy 2012; 17(4): 285-291.

14. Hughes T, Rochester P. The effects of proprioceptive exercise and taping on proprioception in subjects with functional ankle instability: a review of the literature. Physical therapy in sport : official journal of the Association of Chartered Physiotherapists in Sports Medicine 2008; 9(3): 136-147.

15. McKeon PO, Hertel J. Systematic review of postural control and lateral ankle instability, part II: is balance training clinically effective? Journal of athletic training 2008; 43(3): 305-315.

16. Mitchell A, Dyson R, Hale T, Abraham C. Biomechanics of ankle instability. Part 2: Postural sway-reaction time relationship. Medicine and science in sports and exercise 2008; 40(8): 1522-1528.

17. Osborne MD, Chou LS, Laskowski ER, Smith J, Kaufman KR. The effect of ankle disk training on muscle reaction time in subjects with a history of ankle sprain. The American journal of sports medicine 2001; 29(5): 627-632.

18. Willems T, Witvrouw E, Verstuyft J, Vaes P, De Clercq D. Proprioception and Muscle Strength in Subjects With a History of Ankle Sprains and Chronic Instability. Journal of athletic training 2002; 37(4): 487-493.

19. Kim KJ. Impact of Combined Muscle Strength and Proprioceptive Exercises on Functional Ankle Instability. J Int Acad Phys Ther Res 2013; 4(2): 600-604.

20. Sawkins K, Refshauge K, Kilbreath S, Raymond $J$. The placebo effect of ankle taping in ankle instability. Medicine and science in sports and exercise 2007; 39(5): 781-787.

21. Kim KJ, Jegal H, Jun HJ, Choi BJ, Choi HJ, Yu $\mathrm{SH}$, et al. The comparison of balance using Cumberland ankle instability tool to stable and instability ankle. Korean Soc Phys Med 2013; 8(3): 361-368.

22. Schiftan GS, Ross LA, Hahne AJ. The effectiveness of proprioceptive training in preventing ankle sprains in sporting populations: a systematic review and meta-analysis. Journal of science and medicine in sport / Sports Medicine Australia 2015; 18(3): 238-244.

23. Gehring D, Wissler S, Lohrer H, Nauck T, Gollhofer A. Expecting ankle tilts and wearing an ankle brace influence joint control in an imitated ankle sprain mechanism during walking. Gait \& posture 2014; 39(3): 894-898.

24. Higgins M. Therapeutic Exercise: From theory to practice, Philadelphia, Pennsylvania. FA Davis 2011: 273-287.

25. Switlick T, Kernozek TW, Meardon S. Differences in joint-position sense and vibratory threshold in runners with and without a history of overuse injury. Journal of sport rehabilitation 2015; 24(1): 6-12.

26. Lephart SM, Pincivero DM, Giraldo JL, Fu FH. The role of proprioception in the management and rehabilitation of athletic injuries. The American journal of sports medicine 1997; 25(1): 130-137.

27. Cruz-Diaz D, Hita-Contreras F, Lomas-Vega R, Osuna-Perez MC, Martinez-Amat A. Cross-cultural adaptation and validation of the Spanish version of the Cumberland Ankle Instability Tool (CAIT): an instrument to assess unilateral chronic ankle instability. Clinical rheumatology 2013; 32(1): 91-98.

28. Cronkey J, LaPorta G. Rating systems for evaluation of functional ankle instability: prospective evaluation in a cohort of patients treated with monopolar capacitive-coupled radiofrequency. Foot \& ankle specialist 2012; 5(5): 293-299.

29. O'Driscoll J, Kerin F, Delahunt E. Effect of a 6week dynamic neuromuscular training programme on ankle joint function: A Case report. Sports medicine, arthroscopy, rehabilitation, therapy \& technology : SMARTT 2011; 3: 13.

30. Arnold BL, Wright CJ, Ross SE. Functional ankle instability and health-related quality of life. Journal of athletic training 2011; 46(6): 634-641. 